\title{
Annotation
}

\section{Genetic registers}

Two-thirds of couples referred for genetic advice after the birth of an affected child were deterred from having further children when given high recurrence risks (greater than 1 in 10 , and usually 1 in 2 or 1 in 4). ${ }^{1}$ Thus in a sense, genetic counselling can prevent genetic disease by birth control; but there are also more opportunities for prenatal diagnosis and selective abortion, which can lead to compensatory birth of unaffected children. About $85 \%$ of relatives deemed to be at high risk of having a child with a serious genetic disorder have never been given counselling and are unaware of the risks, ${ }^{2}$ and therefore genetic registers designed to improve ascertainment and follow-up of these individuals so that they may be offered genetic counselling, seem a reasonable idea.

In 1977 the Committee of the Clinical Genetics Society formed a Working Party in order specifically to consider: the nature of genetic registers, their value and potential application particularly with regard to the prevention of genetic disease; the place of registers for specific genetic disorderssuch as Huntington's chorea-and genetic disorders in general where relatives may be at high risk; and the feasibility and acceptability of genetic register systems organised either nationally or locally. The chairman, Professor A. E. H. Emery, has just produced his report. ${ }^{3}$

\section{Existing registers}

At present 38 centres in Britain maintain registers primarily concerned with genetic disease so, in practice, the Working Party considered the direction in which we should go rather than whether or not we have genetic registers at all. Existing registers were designed with different functions in mind, and range from not much more than a simple refinement of normal medical records to what the Working Party calls preventive registers designed to improve the ascertainment and follow-up of individuals at risk of transmitting a serious genetic disorder to their offspring, so that they may be offered genetic counselling, including prenatal diagnosis if possible.

It is preventive registers and, to some extent, the large reference research registers that will have far reaching implications for the public and the medical profession. The report outlines three existing computer-aided registers as examples. The RAPID (Register for the Ascertainment and Prevention of Inherited Disease) system was first established in Edinburgh in 1970 and much of what is recommended by the Working Party seems based on this system. The Indiana MEGADAT (Medical Genetics Acquisition and Data Transmission) system collects and processes information on families with genetic disorders at the rate of 1500 per year, and by 1975 had already incorporated data on one-third of a million individuals. The Belgian National Register contains data on all persons (about 6000 a year) referred to any of the seven Belgian medical genetic centres. The last two are very large, multipurpose registers not planned or recommended for this country, and so debate will centre on the recommendations for preventive registers.

\section{Approaching relatives}

A particular problem with the establishment and use of genetic registers (dealing largely with dominant and $\mathrm{X}$-linked recessive diseases) is that diagnosis of the disorder immediately generates important information on relatives who themselves have not sought medical advice. This creates the problem of how to approach family members. The index case has the right to refuse disclosure of his diagnosis to his relatives. Indeed a proportion will express this desire, but in his initial request the doctor may have already intimated that withholding permission to approach relatives could mean unnecessary suffering in the family. The patient now has this extra burden of responsibility to add to his problems. In their discussion of this and similar issues, the Working Party rightly concentrates on the role of the family doctor, so that relatives at high risk are contacted only with the express permission of both the index case and the relative's family doctor, through whom it is best to establish contact. Paediatricians and other specialists will also undoubtedly become involved, and if the activities related to the register are seen to be clearly within the general bounds of family health care, then the majority of families are likely to welcome both the approach and the subsequent counselling. I think it was this sort of sentiment that made the Working Party favour a regional rather than a national register. Access to information in the 
register can, with care, be regulated and limited appropriately; what is much more difficult is to safeguard the interests of those families whose beliefs differ from 'popular' opinion. I believe the tide of prenatal diagnosis is already claiming a few who would have liked to have thought about it longer.

\section{Recommendations of the Clinical Genetics Society Working Party}

The Working Party make the following recommendations:

(1) Registers should be set up for the express purpose of tracing, following-up, and counselling individuals who are at high risk (greater than 1 in 10) of transmitting a serious genetic disorder to their offspring. The object is to ensure that such individuals are informed of the risks and the various options available to them.

(2) Registers should be organised on a 'regional' rather than a national basis, and should be located in a regional genetics centre. If registers already exist in units dealing with specific genetic disorders-such as haemophilia-there should be close liaison with the regional genetics centre in order to provide comprehensive facilities for counselling, including risk estimation.

(3) No individuals should be included on the register without their knowledge and approval.

(4) The involvement of family doctors should be sought in every case and encouraged at all stages of contacting, counselling, and follow-up of individuals at risk.

(5) The complexity of the data and the need for cooperation between centres requires a standard form for recording data and an agreed system for coding disease entities. This system should accommodate chromosomal, multifactorial, and unifactorial disorders and should be sufficiently flexible to include newly recognised genetic disorders and possible heterogeneity.

(6) Computerisation of data in a genetic register is recommended for several reasons. It becomes important for ease of storage and retrieval of data (including patient recall) if large numbers are involved. It has the advantage that, with appropriate safeguards, it helps to maintain strict confidentiality. It also provides a facility for fulfilling other functions such as complex risk calculations.
(7) The computer system for a genetic register should ideally have a dedicated minicomputer located in the genetic centre. The main advantages are increased confidentiality and facility of operation. A less satisfactory alternative is a terminal associated with a large central computer.

(8) There should be standardisation of computer equipment and computer programs, both for economic reasons and to facilitate collaboration between different genetic centres. Therefore, it is essential to co-ordinate developments in this field.

(9) The computer program, like all other aspects of the genetic register system, must incorporate strict safeguards for confidentiality.

(10) Access to data in the register must be restricted to certain clearly specified individuals. Personal and medical information should be released only by the clinician in charge of the register.

(11) In establishing a 'regional' genetic register, provision must be made for adequate supporting staff, which might reasonably include a medical geneticist, a clerk/computer operator, and a field worker.

(12) If a general genetic register is not immediately feasible for economic or other reasons, a limited register should be established for specific genetic disorders of particular importance.

It is desirable that there should be as wide a debate as possible both in and outside the profession before these recommendations are implemented in full. Who is going to monitor the success or failure of this important venture?

\section{References}

${ }^{1}$ Carter, C. O., Roberts, J. A. F., Evans, K. A., and Buck, A. R. (1971). Genetic clinic. A follow-up. Lancet, 1, 281285.

${ }^{2}$ Emery, A. E. H., and Smith, C. (1970). Ascertainment and prevention of genetic disease. British Medical Journal, 3, 636-637.

${ }^{3}$ Emery, A. E. H., Brough, C., Crawfurd, M., Harper, P., Harris, R., and Oakshott, G. (1978). A report on genetic Registers. Based on the Report of the Clinical Genetics Society Working Party. Journal of Medical Genetics, 15, $435-442$.

Marcus E. Pembrey Paediatric Research Unit, The Prince Philip Research Laboratories, Guy's Tower, St Thomas Street, London SE1 9RT 